\title{
MAPEAMENTO DOS FATORES QUE INIBEM A IMPLEMENTAÇÃO DA CULTURA DO E- COMMERCE NO EMPRESARIADO PRUDENTINO
}

\author{
Nilmaer Souza da Silva, Érika Mayumi Kato Cruz, Daiane Almeida Pessoa, Mateus da Silva Adriano. \\ Universidade do Oeste Paulista - Unoeste, Curso de Administração, Presidente Prudente, SP. E_mail: \\ nilmaer@unoste.br
}

\section{RESUMO}

O presente estudo buscou identificar os motivos que desencorajam empresários prudentinos a adotarem o e-commerce e relacionar se as respostas são aderentes ao que se encontra na literatura. A metodologia empregada foi a pesquisa explicativa, com abordagem quantiqualitativa. A coleta de dados pautou-se na pesquisa bibliográfica e na aplicação de questionário estruturado online. A definição da amostra foi por conveniência. A análise de dados deu-se por planilhas eletrônicas, gráficos e análise de conteúdo, proveniente das entrevistas. Os resultados demonstram maior congruência entre a literatura e as respostas dos entrevistados em relação aos fatores estimulantes à adoção do e-commerce do que em relação os elementos inibidores da adoção do e-commerce, revelando respostas ainda não mapeadas pelos pesquisadores. Concluise, que há dois pontos chaves a serem trabalhados em futuras pesquisas, que são a difusão do conhecimento das operações do e-commerce ao empresariado regional e a maximização de mecanismos de segurança virtual.

Palavras-chave: E-Commerce. Comércio Eletrônico. Tecnologia. Organização. Redução de Custos.

\section{MAPPING OF THE FACTORS THAT INHIBIT THE IMPLEMENTATION OF E-COMMERCE CULTURE IN THE PRUDENTINO ENTREPRENEUR.}

\begin{abstract}
The present study sought to identify the reasons that discourage prudent entrepreneurs to adopt e-commerce and to relate if the answers are adherent to what is found in the literature. The methodology used was the explanatory research, with quantitative-qualitative approach. Data collection was based on bibliographic research and the application of a structured questionnaire online. The definition of the sample was for convenience. Data analysis was based on spreadsheets, graphs and content analysis, from interviews. The results demonstrate a greater congruence between the literature and respondents' responses to factors that stimulate the adoption of e-commerce than in relation to the inhibitory elements of e-commerce adoption, revealing responses not yet mapped by the researchers. It is concluded that there are two key points to be worked out in future research, which are the dissemination of knowledge of ecommerce operations to regional entrepreneurship and the maximization of virtual security mechanisms.
\end{abstract}

Keywords: E-Commerce. Electronic Commerce. Technology. Organization. Cost Reduction. 


\section{INTRODUÇÃO}

Desde os últimos anos a internet vem se mostrando uma valiosa e eficiente ferramenta para a geração de capital, viabilizando diversos tipos de negócios, oferecendo uma grande diversidade de possibilidades para o empreendedor e facilidades de compras sob a óptica do consumidor. A internet é um canal promissor, pois reduz custos, facilita a divulgação das empresas e reduz custos operacionais, facilitando assim a venda de produtos e serviços, melhorando até mesmo a relação entre fornecedores e clientes (DIAS, 2003). No Brasil, o comércio eletrônico (ecommerce), que é definido por Moreira (2016) como um ambiente de compra e venda de produtos e serviços por meio da internet, vem crescendo muito, de forma democrática e transversal com relação às classes sociais, pois quase $80 \%$ da população brasileira já fez alguma compra online, o que impulsiona investimento de empreendedores nesse processo (VAVILIS; PETKOVIC; ZANNONE, 2014; TEIXEIRA, 2015; CRUZ, et al., 2017; SERRENTINO, 2017).

Do lado do consumidor, o e-commerce viabiliza compras de qualquer lugar do planeta, com entrega no local desejado, de forma fácil, prática, rápida, conveniente e em certa medida segura, permitindo, ainda, ampla comparação de preços (MOREIRA, 2016; MAZETO, 2018). Para o empreendedor, o comércio eletrônico também se revela apropriado e atraente, pois, praticamente, inexistem barreiras geográficas, dispensam, por vezes, lojas físicas, tornando-se o site o seu ponto de venda, reduzindo, de conseguinte, os seus custos operacionais com pessoas, locação, dentre outros (COELHO; OLIVEIRA; ALMÉRI, 2013; CEZAR, et. al., 2014; COSTA, 2017). Em outra perspectiva, o e-commerce ainda gera insegurança e preocupações para usuários e empreendedores, em virtude dos riscos de compras pela internet em função das falsificações de sites, propostas e anúncios falsos, sendo esses crimes, ainda frequentes (ANDRADE; SILVA, 2017). Os crimes virtuais como fraudes, sabotagens, espionagens, dentre outros crimes cibernéticos são tipificados na Lei $12737 / 2012^{1}$. Todavia, esses crimes virtuais, incluindo-se as clonagens de cartões e dados pessoais que geram prejuízos às pessoas e organizações causam medo e insegurança nos usuários de internet e nos empreendedores (PAIXÃO; CAETANO; ALVARENGA, 2005).

Ainda que seja um ambiente estratégico para divulgação de produtos, serviços e de inovações, segundo Bastos, Feldmann e Fouto (2014) as dificuldades para criação de uma plataforma segura, intuitiva e de fácil interação com o usuário, consultorias inexperientes, falhas em projetos, investimentos vultosos em tecnologia da informação - TI, logística de distribuição de produtos e logística reversa são elementos inibidores da adoção do e-commerce por alguns empreendedores (ARANHA, 2010; ZILBER, 2016; FUMAGALLI; BOTTAMELLI; RAZÕES, 2016).

Nessa perspectiva, o presente estudo teve como objetivo precípuo, identificar os motivos que desencorajam alguns empresários da cidade de Presidente Prudente a comercializarem os seus produtos via e-commerce e relacionar se os motivos alegados por estes são aderentes ao que se encontra na literatura.

\section{METODOLOGIA}

Trata-se de pesquisa explicativa, com abordagem quanti-qualitativa, cujos instrumentos de coleta de dados foram a pesquisa bibliográfica e o questionário estruturado online, sem possibilidade de identificação ulterior dos pesquisados, valendo-se, para tanto, da amostragem por conveniência. A análise dos dados ocorreu por meio de planilhas eletrônicas, gráficos e, por fim, análise de conteúdos, comparando a bibliografia utilizada com as respostas dos entrevistados.

\footnotetext{
${ }^{1}$ Lei no 12.737/2012 trata sobre a tipificação criminal de delitos informáticos. Disponível em: http://www.planalto.gov.br/ccivil 03/ ato20112014/2012/lei/l12737.htm. Acesso em 18 de maio de 2018.
} 


\section{RESULTADOS}

Foram entrevistados 20 empresários, sem identificação de idade e outros traços demográficos que pudessem caracterizar a identificação dos sujeitos da pesquisa. Os empreendedores, no entanto, atuam nos ramos de tecnologia, saúde, consultoria, comércio alimentício e construção civil. $40 \%$ dos entrevistados atuam há mais nove anos. $33 \%$ entre três e oito ano e os demais (27\%) têm suas empresas há dois ou menos anos. $54 \%$ dos respondentes possuem lojas físicas e virtuais. 33\% apenas lojas físicas e $13 \%$ lojas online, apenas. Questionados sobre os fatores que inibem a implementação do e-commerce em suas lojas físicas, responderam o que se segue.

Gráfico 1. Fatores que inibem o uso do e-commerce em empresas físicas.

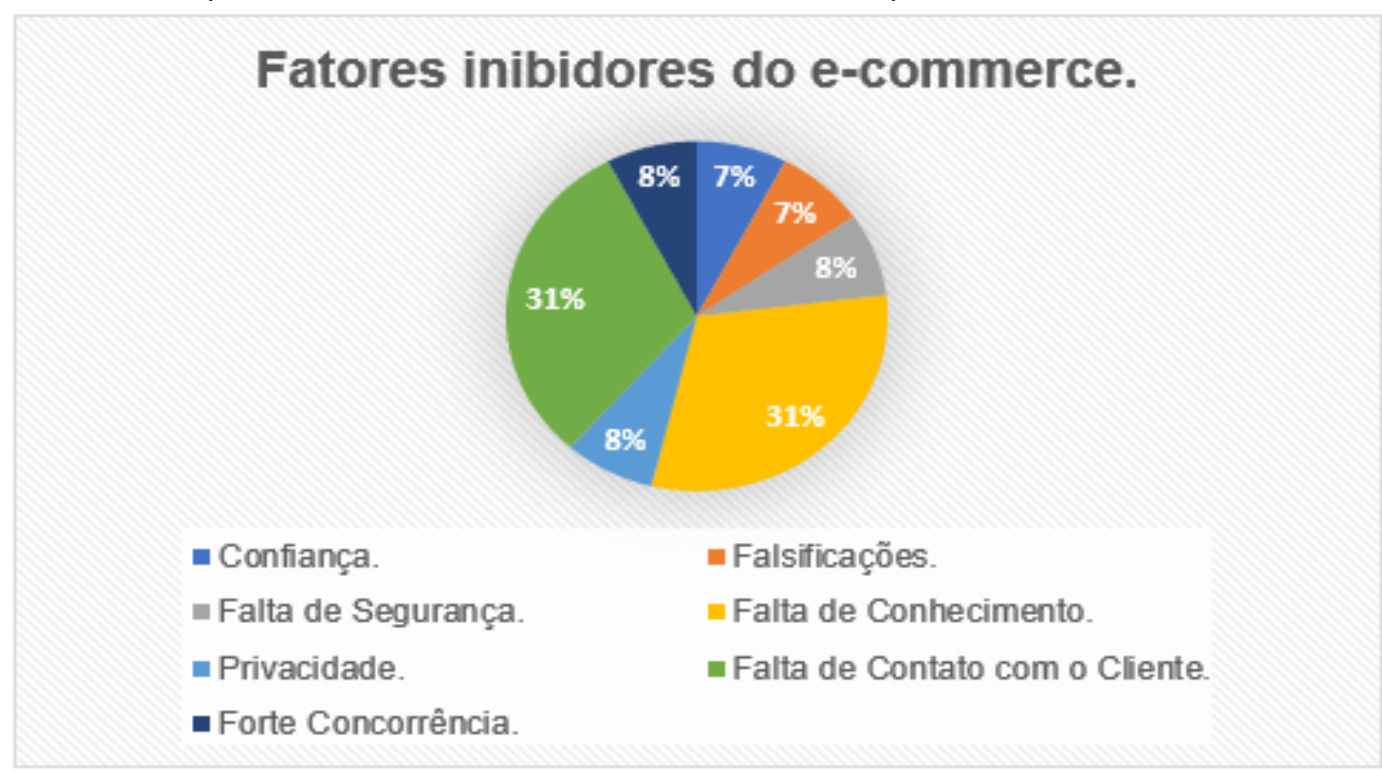

Fonte: Elaborado pelos autores.

Observa-se o fator inibidor do uso do e-commerce mais evidente é a falta de conhecimento (dos mecanismos de operação do e-commerce). Já acerca dos fatores que motivam a adoção do e-commerce, obtêm-se as respostas pontuadas no gráfico a seguir. 
Gráfico 2. Fatores que motivam a utilizaçãodo e-commerce.

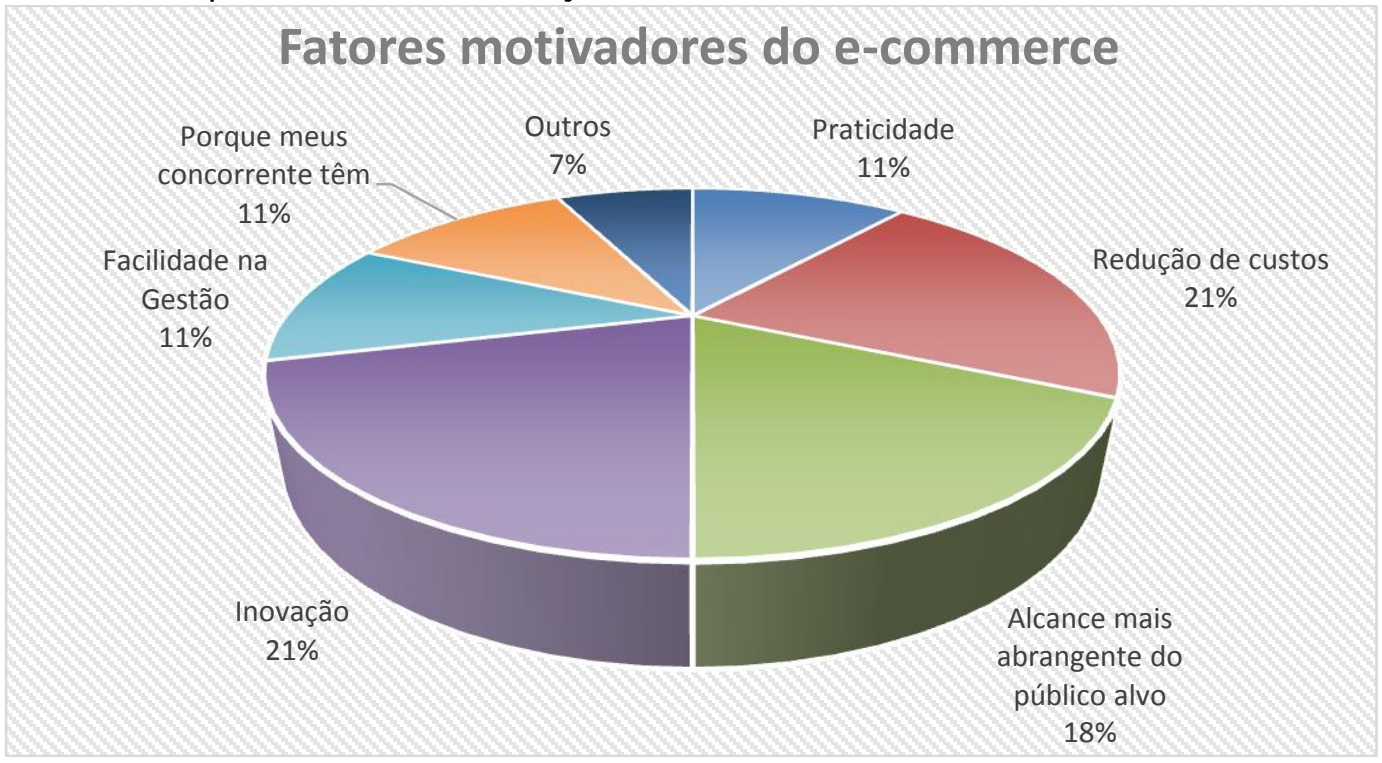

Fonte: Elaborado pelos autores.

Consoante à literatura, a redução de custos, facilidade de gestão, praticidade e inovação são os fatores que estimulam os empreendedores a investirem no comércio eletrônico. Questionados sobre o nível de conhecimento em relação ao e-commerce, 53\% atribuíram peso cinco, numa escala de um a cinco, afirmando conhecer muito bem o comércio eletrônico, mas, apenas na perspectiva da compra. Relatando, indiretamente, que não conhecem as nuances do lado do vendedor. $20 \%$ atribuíram peso quatro e $27 \%$ peso 3 . No tocante a já terem sido vítimas de crimes virtuais, $73,3 \%$ dos respondentes alegaram já terem sido vítima, ao menos uma vez, de crimes virtuais.

\section{DISCUSSÃO}

Os empreendedores têm buscado investir e obterem vantagens competitivas por meio do e-commerce por diversos motivos. O presente estudo revela coerência entre os elementos motivadores do investimento no comércio eletrônico entre o que propõe a literatura e o que dizem os entrevistados, sendo eles, especialmente, nas suas próprias palavras, redução de custos, alcance mais abrangente de público - ausência de barreiras geográficas -, facilidade e praticidade na gestão, em linha com o que preconizaram os autores (COELHO; OLIVEIRA; ALMÉRI, 2013; CEZAR, et. al., 2014; COSTA, 2017). No que concerne aos elementos inibidores ou desencorajadores sobre investimento no comércio eletrônico, a falta de conhecimento (operativo) e a ausência de contato com o cliente foram os mais citados pelos respondentes da pesquisa, $31 \%$ para ambas as respostas. Esses elementos refletem respostas livres dos pesquisados, ou seja, sem indicação de um rol de possibilidades pelos autores, isto é, fatos escolhidos per ser expressão de limitações oriundas de autoavaliação. Ademais, essas afirmações não foram mapeadas na literatura correlata, isto é, previstas pelos autores empregados neste estudo. Os elementos citados pelos autores, Aranha (2010), Zilber (2016), Fumagalli, Bottamelli e Razões, (2016), tais como as dificuldades para criação de uma plataforma segura, intuitiva e de fácil interação com o usuário, consultorias inexperientes, falhas em projetos, investimentos vultosos em tecnologia da informação - TI, logística de distribuição de produtos e logística reversa não foram citados pelos entrevistados. Isso revela, em certa medida, aspectos educacionais, informacionais e culturais que carecem de serem tratados junto ao empresariado objeto da pesquisa. Aparece, no entanto, de forma menos expressiva, nas respostas dos entrevistados, o que preconizou Andrade e Silva (2017), quando argumentam que dos maiores aspectos inibidores da adoção do e-commerce é são os riscos cibernéticos. O comércio eletrônico, porém, no mundo todo já se tornou uma realidade, 
devido à facilidade e praticidade que este mecanismo de compra oferece aos consumidores na hora da aquisição e aos empreendedores na hora de comercializar e vender seus produtos e serviços pela internet (COELHO; OLIVEIRA; ALMÉRI, 2013). Ainda nessa enredo, Oliveira e Alméri (2013) afirmam que os maiores motivos para se investir no comércio eletrônico são os amplos acessos às informações do mercado, de preços, de produtos, formas de vender, serviços e recursos disponíveis, o que gerou aos empreendedores muitas inovações na maneira de comercializar produtos e serviços de forma prática e sem muitos gastos.

\section{CONCLUSÃO}

Em um cenário em que a tecnologia vem crescendo rapidamente dia após dia, as empresas estão se adaptando às mudanças, para conseguirem se manter competitivas no mercado. Contudo, ainda existem muitos aspectos negativos e obstáculos a serem superados que desencorajam os empresários a investir no comércio eletrônico, e para isso, é necessário que o conhecimento sobre o e-commerce se torne cada mais vez elevado e conheçam as vantagens que esta ferramenta proporciona, como o baixo custo, redução do tempo, satisfação do cliente, maior qualidade, pois atualmente no Brasil, o e-commerce vêm tendo um grande sucesso já que $80 \%$ da população brasileira optam em realizar compras online. Dados os limites da pesquisa, local, não é possível concluir se a ausência de conhecimento sobre os mecanismos operativos do e-commerce e outros fatores inibidores da sua adoção refletem a verdade regional, estadual ou nacional. Assim, há uma importante oportunidade de aprofundamento e continuidade deste estudo em maior abrangência. Fato é que ações extensionistas de Universidades, serviços oferecidos por consultorias e instituições de apoio ao empreendedor, como o SEBRAE, por exemplo, podem servir de apoio a esses empresários, de modo a impulsionar a adoção do e-commerce. Dessa forma, há dois pontos chaves a serem trabalhados em futuras pesquisas, quais sejam: difusão do conhecimento das operações do e-commerce ao empresariado regional e maximização de mecanismos que gerem segurança no ambiente virtual.

\section{REFERÊNCIAS}

ANDRADE, M. C. F.; SILVA, N. T. G. O Comércio Eletrônico (E-Commerce): Um Estudo com Consumidores. Perspectivas em Gestão \& Conhecimento, v. 7, n. 1, p. 98-111, 2017. Disponível em: http://www.spell.org.br/documentos/ver/45744/o-comercio-eletronico--e-commerce---umestudo-com-consumidores/i/pt-br Acesso em: 24 mar. 2018.

ARANHA, H. S. Fatores Inibidores à adoção de tecnologia de informação em micro e pequenas empresas fornecedoras da Vale no estado do Pará. Dissertação apresentada ao programa de pósgraduação em administração da universidade federal do rio grande do norte. Natal, jul. 2010. Disponível em: https://repositorio.ufrn.br/jspui/bitstream/123456789/12178/1/HelderSA DISSERT.pdf $\quad$ Acesso em: 01 mai. 2018.

BASTOS, L. T.; FELDMANN, P. R.; FOUTO, N. M. M. D. Inovação de varejo sustentável: E-commerce e comércio justo. Revista de Administração da UFSM, v. 7, n. esp, p. 88-99, 2014.

CEZAR, S. et. al. Efeitos do Comércio Eletrônico: a percepção dos empresários associados à Câmara de Dirigentes Lojistas (CDL) de São Miguel do Oeste-SC. Navus - Revista de Gestão e Tecnologia. Florianópolis, SC, v. 4, n. 1, p. 111-125, jan./jun. 2014. Disponível em: http://navus.sc.senac.br/index.php/navus/article/download/172/146 Acesso em: 02 mai. 2018. 
COELHO, L. S., Oliveira, R. C. \& Alméri, T. M. O CRESCIMENTO DO E-COMMERCE E OS PROBLEMAS QUE O ACOMPANHAM: a identificação da oportunidade de melhoria em uma rede de comercio eletrônico na visão do cliente. Revista de Administração do UNISAL. Campinas, v. 3, n. 3, p. 63-85, Jan/Abr 2013.

Disponível

em: <http://www.revista.unisal.br/sj/index.php/RevAdministracao/article/download/235/202/0> Acesso em: 01 mar. 2018.

COSTA, L. S. Análise do nível de aceitação do e-commerce de acordo com a percepção dos discentes de administração e marketing de uma faculdade situada no centro-oeste de Minas Gerais. Formiga: UNIFOR/MG, 2017. 43 p. (Trabalho de Conclusão de Curso, Superior de Tecnologia em Marketing). Disponível em:https://repositorioinstitucional.uniformg.edu.br:21074/xmlui/handle/123456789/444 Acesso em: 24 mar. 2018.

CRUZ, L.A.M, et al. Desenvolvimento do E-commerce no Brasil. 02 jan. 2017. Disponível em: http://www.administradores.com.br/artigos/academico/o-desenvolvimento-do-e-commerce-nobrasil/101304/ Acesso em: 17 mar. 2018.

DIAS, D. A. S. Avaliação da Adoção de Soluções E-Bussiness por Empresas Brasileiras. São Paulo: EAESP/FGV, 2003. 130 p. (Dissertação de Mestrado apresentada ao curso de Pós-Graduação da FGV/EAESP, Área de Concentração: Mercadologia). Disponível em: http://hdl.handle.net/10438/2218 Acesso em 17 mar. 2018.

FUMAGALLI, L. A. W; Bottamelli, K; Razões, R. S. P. Os desafios da indústria no processo de atendimento ao consumidor por meio do comércio eletrônico. Campo Grande, v. 4, n. 3, p. 01-14, set/dez., 2016. Disponível em: http://www.desafioonline.com.br/publicações Acesso em: 01 mai. 2018.

MAZETO, T. 7 Motivos que levam ao crescimento do mercado eletrônico no Brasil. ECommercebrasil: excelência em e-commerce. 23 mar. 2018. Disponível em: https://www.ecommercebrasil.com.br/artigos/crescimento-do-mercado-eletronico/ Acesso em 24 mar. 2018.

MOREIRA, R. A. O Comércio Eletrônico, os Métodos de Pagamentos e os Mecanismos de Segurança. REFAS - Revista Fatec Zona Sul, v.3, n. 1, p. 15, 2016. Disponível em: http://www.revistarefas.com.br/index.php/RevFATECZS/article/view/67 Acesso 17 mar. 2018.

PAIXÃO, L. A; Caetano, M. N; Alvarenga, C. S. CRIMES CIBERNÉTICOS: EVOLUÇÃO DO DIREITO PENAL ELETRÔNICO FRENTE ÀS NOVAS DEMANDAS DA VIDA ATUAL. Revista Juridica Da, n.1, p. 11, 2005. Disponível em: http://www.egov.ufsc.br/portal/sites/default/files/artigo3.pdf Acesso em: 17 mar. 2018.

SERRENTINO, A. Brasil, Comércio Eletrônico e Transformação Digital. Artigos Conteúdo ONDV. 05 out. 2017. Disponível em: http://onegociodovarejo.com.br/brasil-comercio-eletronico-etransformacao-digital/ Acesso em: 24 mar. 2018.

TEIXEIRA, TARCISIO. Comércio Eletrônico: Conforme o Marco Civil da Internet e a regulamentação do e-commerce no Brasil. São Paulo: Saraiva,2015. 
VAVILIS, S., Petkovic, M., \& Zannone, N. (2014). A reference model for reputation systems.Decision Support Systems, 61(1), 147-154. doi: 10.1016/j.dss.2014.02.002. https://doi.org/10.1016/i.dss.2014.02.002

ZILBER, S. N.O papel da área de TI na implantação de comércio eletrônico: o caso da Ford do Brasil. Revista de Administração e Inovação, v. 3, n. 2, p. 47-61, 2006. 